\title{
AuVeg - a database of German floodplain vegetation
}

\author{
Peter Joerg Horchler, Eva Mosner \& Jan Peper
}

\begin{abstract}
AuVeg (GIVD ID EU-DE-028) is a database containing phytosociological relevés of German floodplain vegetation including key environmental variables such as hydrological and soil data. It provides essential information to further understand ecological interactions in floodplain habitats. Furthermore, it provides baseline data for the development of habitat models of plant species and vegetation types. Such models will be used to assess the effect of climate change-induced alterations of the riverine flow regime on plant habitats. They are also used in practice supporting the environmental impact assessment in case of riverine construction or maintenance works.
\end{abstract}

Keywords: habitat model; hydrology; phytosociology.

\section{AuVeg}

Scope: Database of German floodplain vegetation including environmental variables

Status: emerging

Period: $1981-2011$

Database manager(s): Peter Joerg Horchler (horchler@bafg.de); Eva Mosner (mosner@bafg.de); Jan Peper (peper@bafg.de)

Owner: Federal Institute of Hydrology

Web address: [NA]

Availability: not yet available

Database format(s): TURBOVEG

Online upload: no Online search: no

Publication: [NA]

Plot type(s): normal plots

Non-overlapping plots: 5,180

Total plot observations: 5,180

Countries: DE: $100.0 \%$

Forest: 29\% - Non-forest: aquatic: $2 \%$; semi-aquatic: 19\%; arctic-alpine: 0\%; natural: $10 \%$; semi-natural: $31 \%$; anthropogenic: $10 \%$

Guilds: all vascular plants: $100 \%$

Environmental data: altitude: $100 \%$; slope aspect: $5 \%$; slope inclination: $5 \%$; soil depth: $5 \%$; surface cover other than plants (open soil, litter, bare rock etc.): $15 \%$; soil pH: $1 \%$; other soil attributes: $100 \%$

Performance measure(s): cover: $95 \%$; other: $5 \%$

Geographic localisation: GPS coordinates (precision $25 \mathrm{~m}$ or less): $90 \%$; point coordinates less precise than GPS, up to $1 \mathrm{~km}: 10 \%$

Sampling periods: $1980-1989$ : $2.0 \%$; 1990-1999: 53.0\%; 2000-2009: 30.0\%; 2010-2019: $10.0 \%$

Information as of 2012-07-12; further details and future updates available from http://www.givd.info/ID/EU-DE-028

Peter Joerg Horchler* (horchler@bafg.de), Eva Mosner (mosner@bafg.de), Jan Peper (peper@ bafg.de)

Ecological Interactions, Federal Institute of Hydrology, Am Mainzer Tor 1, 56068 Koblenz, GERMANY

*Corresponding author 